\title{
Der Sanktionsrabatt im abgekürzten Verfahren
}

In der wissenschaftlichen Diskussion über das abgekürzte Verfahren wird das zwischen den Parteien ausgehandelte Strafmass zuweilen vernachlässigt. Im Mittelpunkt steht dabei die Frage, wann und in welchem Umfang sich hier ein Sanktionsrabatt rechtfertigen lässt. Die vertiefte Erörterung dieser Thematik führt im Ergebnis zur Forderung eines Verzichts auf die in der Praxis gewährten grosszügigen Strafminderungen. Von hier aus kann man sogar noch einen Schritt weiter gehen und die kriminalpolitische Legitimation des gesetzlich geregelten Abspracheverfahrens insgesamt in Zweifel ziehen.

I. Der typische Rabatt

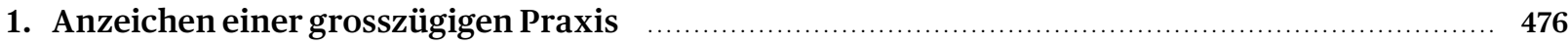

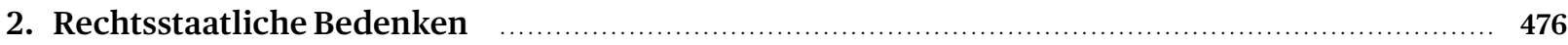

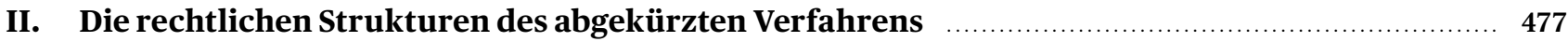

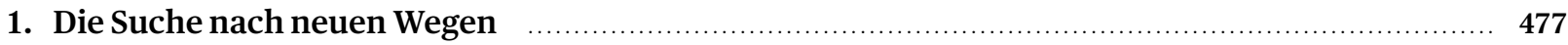

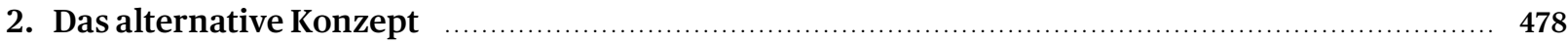

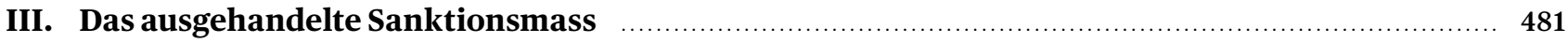

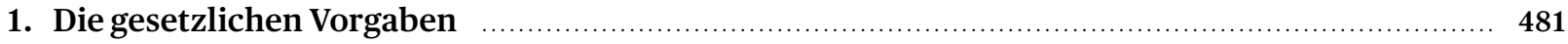

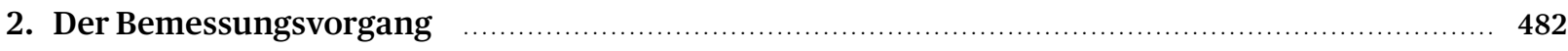

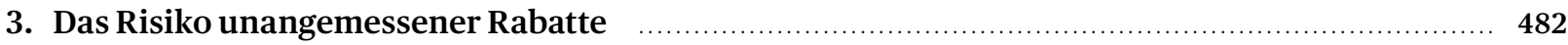

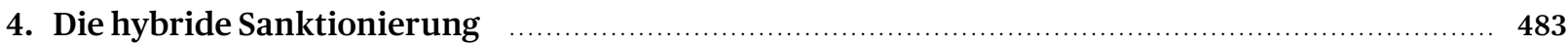

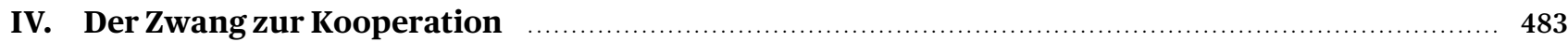

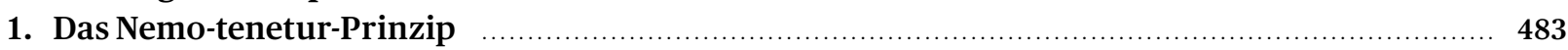

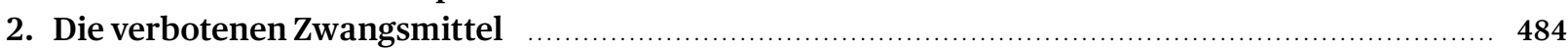

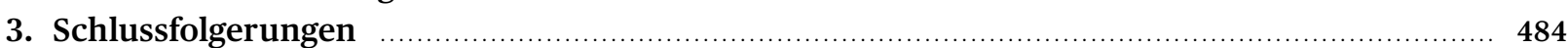

Zitiervorschlag:

PETER ALBRECHT, Der Sanktionsrabatt im abgekürzten Verfahren, sui generis 2020, S. 475

Prof. Dr. Peter Albrecht, emeritierter Extraordinarius für Strafrecht und Strafverfahrensrecht an der Universität Basel und ehemaliger Strafgerichtspräsident in Basel.

URL: sui-generis.ch/158

DoI: https://doi.org/10.21257/sg.158

Dieses Werk ist lizenziert unter einer Creative Commons Namensnennung - Weitergabe unter gleichen Bedingungen 4.0 International Lizenz. 


\section{Der typische Rabatt}

\section{Anzeichen einer grosszügigen Praxis}

1 Die gesetzlichen Vorschriften des abgekürzten Verfahrens (Art. 358 ff. StPO1) vermitteln in ihrer praktischen Anwendung nach aussen hin vor allem den generellen Eindruck verhältnismässig tiefer Strafen. In solchen Prozessen findet zwischen den Parteien regelmässig eine Art Tauschhandel statt, vereinfacht ausgedrückt: eine Strafminderung im Austausch gegen ein Geständnis. Das kooperative Verhalten der beschuldigten Personen erfährt nämlich mittels einer Absprache hinsichtlich des Strafmasses typischerweise ${ }^{2}$ eine grosszügige Honorierung, und zwar nicht selten über die Grenzen der zentralen Vorschrift des Art. 47 StGB$^{3}$ hinaus. Daraus kann leicht eine weiter reichende Sanktionsminderung als bei der Beurteilung geständiger Beschuldigter im ordentlichen Verfahren resultieren. Folglich liegt es nahe, hier plastisch von einem Sanktionsrabatt ${ }^{4}$ zu sprechen. Dessen Berechtigung wird jedoch im juristischen Schrifttum unter anderem deshalb angezweifelt, weil damit die Tendenz zu unverantwortlich milden Sanktionen verbunden sei. 5

\section{Rechtsstaatliche Bedenken}

2 Das hier vorangestellte Bild der Rechtsprechung ist zunächst bewusst kurz und bloss in groben Umrissen skizziert worden. Doch bereits ein erster Blick darauf löst sogleich rechtsstaatliche Verunsicherungen aus. Der Gesetzgeber hat nämlich mit dem durch die schweizerische Prozessordnung eingeführten abgekürzten Verfahren (ziemlich unbekümmert) fundamentale Rechtsprinzipien infrage gestellt. Gegen das auf Absprachen zwischen den Parteien zugeschnittene abgekürzte Verfahren sind immer wieder ganz grundsätzliche Einwände erhoben worden. Niklaus Oberholzer etwa äusserte sich schon im Jahr 1992 (erstmals für das schweizerische Recht) dezidiert kritisch gegen die vereinbarten Verfah-

1 Schweizerische Strafprozessordnung vom 5. Oktober 2007 (StPO; SR312.0).

2 Instruktiv diesbezüglich der Titel der Dissertation von EMANUEL JAGGI, Die prototypische Absprache, Bern 2006.

3 Schweizerisches Strafgesetzbuch vom 21. Dezember 1937 (StGB; SR 311.0).

4 Dieser Begriffbringt zum Ausdruck, dass der vorliegende Text sich auf die Unterschiede zwischen dem ordentlichen und dem abgekürzten Verfahren im Strafmass (d.h. in der Strafhöhe) fokussiert.

5 Zum Ganzen ANGELA GIGER, Die Unverwertbarkeit der Erklärungen nach Art. 362 Abs. 4 StPO: Rückschluss auf das Strafmassermessen der Staatsanwaltschaft im abgekürzten Verfahren? - Besprechung von BGE144 IV 189, fp 2019, S.74, mit zahlreichen Nachweisen; ferner NIKLAUS SCHMID / DANIEL JOSITSCH, Schweizerische Strafprozessordnung (StPO), Praxiskommentar, 3. Aufl., Zürich et al. 2018, Art. $362 \mathrm{~N} 4$. rensbeschleunigungen in der Rechtsprechung: «Absprachen im Strafprozess widersprechen beinahe sämtlichen strafprozessualen Grundsätzen.»6 Man mag zwar gute Gründe finden, die pauschal formulierte Kritik zu relativieren. ${ }^{7}$ Trotzdem erweist sich als überaus bedenklich, dass im abgekürzten Verfahren das Hauptziel der Beschleunigung bzw. Effizienz «alle anderen prozessualen Wertentscheidungen in den Hintergrund rückt».8

Der harte Befund erschüttert das Verfahrensrecht in sei- 3 nen Grundfesten. Ausserdem hat er (zumindest indirekte) Auswirkungen auf das materielle Recht. Es deutet sich ein weitreichender Wandel in den Vorstellungen über Sinn und Zweck kriminalrechtlicher Interventionen an. ${ }^{9}$ Evident wird das insbesondere im Bereich der Strafzumessung. So ist auf dem Boden eines traditionellen Verständnisses von Straf(prozess)recht beispielsweise schwer verständlich, dass zwischen der Staatsanwaltschaft und der beschuldigten Person mittels Absprache eine Strafsanktion (inklusive Rabatt) ausgehandelt werden darf. Wie kann man ein solches «Tauschgeschäft», das auf eine Strafminderung gegen ein Geständnis abzielt, sachlich legitimieren? Wo bleibt da im Übrigen das unabhängige Gericht? ${ }^{10}$

Mit Blick auf die gesetzliche Regelung drängt sich die 4 grundlegende Frage auf, unter welchen Voraussetzungen und in welchem Ausmass sich im abgekürzten Verfahren ein Sanktionsrabatt rechtfertigen lässt. Dieser Thematik widmet sich die vorliegende Abhandlung. Hierfür richte ich in einem ersten Schritt den Fokus auf zentrale strukturelle Aspekte des Prozessrechts (nachfolgend Ziff. II). Sodann wird der an den Massstäben der Art. $47 \mathrm{ff}$. StGB orientierte Vorgang eines ausgehandelten Strafmasses vertieften Erörterungen unterzogen (Ziff. III). Daraus ergeben sich schliesslich - wie zu zeigen sein wird - über die in den Vordergrund gerückte Problemstellung hinaus ganz prinzipielle Bedenken gegen das Institut des abgekürzten Verfahrens (Ziff. IV).

6 NIKLAUS OBERHOLZER, Informelle Absprachen im Strafverfahren, AJP 1992, S.14; ebenso DERSELBE, Absprachen im Strafverfahren - pragmatische Entlastungsstrategie oder Abkehr vom strafprozessualen Modell?, ZStrR 1993, S.163.

7 Vgl. etwa die differenzierenden Stellungnahmen von WOLFGANG WOHLERS, Fair Trial-Grundpfeiler oder Feigenblatt?, fp 2019, S. 209f., und ADAM EL-HAKIM, Mitbeschuldigte im abgekürzten Verfahren gemäss Art. 358 ff. StPO, Basel 2017, S. 17; ferner SVEN ZIMMERLIN, Der Verzicht des Beschuldigten auf Verfahrensrechte im Strafprozess, Diss. Zürich 2009, N714.

8 FELIX BOMmER, Abgekürztes Verfahren und Plea Bargaining im Vergleich, ZSR 2009 II, S. $27 \mathrm{f}$.

9 Weiterführend hinten Ziff. III.4.

10 Ergänzend dazu hinten Ziff. II.2.b, III.2.a und III.3. 


\section{Die rechtlichen Strukturen des abgekürzten Verfahrens}

\section{Die Suche nach neuen Wegen \\ a) Überlastung der Justiz}

5 Im abgekürzten Verfahren bildet - wie schon die Bezeichnung andeutet - «die verfahrensverkürzende Wirkung Hauptmotiv» jeder Absprache zwischen den Parteien. ${ }^{11}$ Das geschieht als Folge einer regelmässig beklagten «Überlastung der Strafverfolgungsbehörden». ${ }^{12}$ Das Bedürfnis einer Entlastung der Justiz wird namentlich dort als aktuell dargestellt, wo in komplexen Verfahren eine unklare Tatsachen- oder Rechtslage besteht und somit langwierige Beweisaufnahmen mit ungewissem Ergebnis drohen. ${ }^{13}$ Es handelt sich häufig um Situationen, die zusätzlich durch einen Mangel an (personellen) Ressourcen gekennzeichnet sind. Als primäre Beispiele nennen die Gesetzesmaterialien die Bereiche der Wirtschafts- oder Drogenkriminalität. ${ }^{14}$ Dort ermöglicht die geständige Person dem Staat, allenfalls einen gar nicht oder nur schwer durchsetzbaren Strafanspruch zu verwirklichen. Im Alltag dominieren die abgekürzten Verfahren bei sog. opferlosen Delikten, etwa bei Widerhandlungen gegen das Betäubungsmittelgesetz. ${ }^{15}$ Teilweise wird sogar die Prognose gewagt, dass bei dieser Art von Straftaten in absehbarer Zeit das abgekürzte Verfahren das ordentliche verdrängen werde. ${ }^{16}$

6 Immer wieder stösst man auf dramatisierende Äusserungen, wonach die Strafbehörden auf der Grundlage eines ordentlichen Verfahrens nicht mehr in der Lage seien, ihre Aufgabe zu bewältigen; teilweise wird sogar von einem «Ermittlungsnotstand» ${ }^{17}$ gesprochen. Für solche Befunde mangelt es indessen an valablen empiri-

11 OBERHOLZER, Absprachen im Strafverfahren 1993 (Fn. 6), S. 159.

12 Botschaft zur Vereinheitlichung des Strafprozessrechts, BBl 2006 1085, S. 1295; ausführlich dazu MARION LAGLER, Besondere Verfahrensarten: Überlastung der Strafjustiz oder Ausdruck erhöhter Punitivität?, Zürich et al. 2016, S.151ff.

13 NIKLAUS OBERHOLZER, Grundzüge des Strafprozessrechts, 4. Aufl., Bern 2020, N1969.

14 Botschaft zur Vereinheitlichung des Strafprozessrechts (Fn.12) S. 1295; GEORGES GREINER / IRMA JAGGI, in: Niggli/Heer/Wiprächtiger (Hrsg.), Basler Kommentar, Schweizerische Strafprozessordnung, 2. Aufl., Basel 2014, vor Art. 358-362 N21; CHRISTIAN SCHWARZENEGGER, in: Donatsch/Hansjakob/Lieber (Hrsg.), Kommentar zur Schweizerischen Strafprozessordnung (StPO), 2. Aufl., Zürich et al. 2014, Art. 358 N3.

15 In diesem Sinne OBERHOLZER, Grundzüge (Fn.13), N1968 und 1977; BARBARA LIPS-AMSLER, Abgekürzte Verfahren im Wirtschaftsstrafprozess - aus richterlicher Sicht, in: Ackermann/Hilf (Hrsg.), Kurzer Prozess, zu kurzer Prozess - im Wirtschaftsstrafverfahren, Zürich et al. 2019, S.143; STÉPHAN GRODECKI, Expériences pratiques de la procédure simplifiée, fp 2016, S. 48 f.; SCHWARZENEGGER (Fn.14), Art. 358 N2.

16 MARC THOMMEN, Kurzer Prozess - fairer Prozess?, Bern 2013, S. 241

17 E. JAGGi (Fn. 2), S.104 ff. schen Belegen. ${ }^{18}$ Selbst wenn man aber die feststellbare hohe Belastung bzw. Überlastung als Argument für beschleunigte Prozesse anerkennt, bleibt immer noch die Frage, weshalb es gerade die abgekürzten Verfahren gemäss Art. $358 \mathrm{ff}$. StPO braucht. Bringt denn diese Verfahrensart tatsächlich der Justiz die erhoffte Entlastung? Weshalb genügen hierfür nicht etwa die praktisch weitaus bedeutsameren Strafbefehlsverfahren (Art. 352 ff. StPO) ${ }^{19}$ oder (ergänzend) eine Ausweitung des Opportunitätsprinzips (Art. 8 StPO)?

\section{b) «Normeninflation»}

Einzelne Stimmen in der Literatur betonen, dass die kol- 7 portierten Überlastungsklagen auf unterschiedlichen Ursachen beruhen, die unter anderem auch wesentlich das materielle Recht mit seiner permanenten Erweiterung der Verbotsmaterie tangieren. ${ }^{20}$ Das Strafrecht beschränkt sich heute nämlich nicht auf den Schutz elementarer Rechtsgüter, die für den Bestand der Gesellschaft unerlässlich sind. Offenkundig setzt der Staat die Strafe nicht mehr als letztes Mittel ein, sondern immer öfters als bloss symbolisches Steuerungsinstrument zur erhofften Bewältigung von gesellschaftlichen Krisenphänomenen unterschiedlichster Art. Wegleitend sind ziemlich beliebige und teils diffuse ordnungspolitische Bedürfnisse ohne kohärentes kriminalpolitisches Programm. ${ }^{21}$ In rascher Kadenz produziert der Gesetzgeber neue Strafbestimmungen, oder bisherige Tatbestände werden ausgedehnt. Anhand der hervorgehobenen «Normeninflation»"22 lässt sich jedoch nicht schlüssig belegen, dass gerade die neu geschaffenen Tatbestände für die beklagte Überlastung der Strafjustiz kausal sind; die vorliegenden empirischen Daten sprechen eher dagegen. ${ }^{23}$ Offenkundig spielten deshalb für die Einführung des abgekürzten Verfahrens im Hintergrund weitere (nicht explizit genannte) kriminalpolitische Aspekte eine massgebende Rolle. ${ }^{24}$

18 Kritisch auch THOMMEN (Fn.16), S. 257 f., und LAGLER (Fn.12), S.164f.

19 Dies fragt sich umso mehr, als gemäss der Ansicht von NIKLAUS OBERHOLZER, «Die Wahl des Verfahrens und das gerechte Urteil», Plädoyer 5/2015, S.16, das abgekürzte Verfahren heute eigentlich nichts anderes ist «als ein qualifizierter Strafbefehl für das Grobe, der zusätzlich noch der notariellen Bestätigung durch das Gericht bedarf».

20 Z.B. LAGLER (Fn.12), S.151ff., und OBERHOLZER, Absprachen im Strafverfahren 1993 (Fn. 6), S.159ff.

21 PETER ALBRECHT, Strafrecht ohne Recht?, ZStrR 2013, S. 387.

22 NIKLAUS OBERHOLZER, Von der Normeninflation im Strafrecht, in: Riklin/von Däniken (Hrsg.), Straflust oder Straffrust?, Bern 2011, S.11ff.

23 Eindrucksvoll und differenzierend LAGLER (Fn.12), S.151ff.

24 Man mag hier an den Gesichtspunkt der «Punitivität» (einlässlich dargestellt bei LAGLER [Fn.12], S.166ff.) oder auch an das prozessuale Interesse einer Sicherung des Schuldspruchs (dazu hinten Ziff. II.2.c.cc) denken. 
8 Trotz aller Erklärungsversuche erscheint die gesetzliche Regelung des abgekürzten Verfahrens - von aussen her betrachtet - als Produkt eines einseitigen instrumentellen Denkens. Aus dem heraus entwickelt sich ein vordringliches «Effizienzinteresse» ${ }^{25}$ mit entsprechenden Implikationen im Prozessrecht, und zwar weit über die strafmindernde Wirkung des Geständnisses hinaus.

\section{Das alternative Konzept}

\section{a) Beschleunigungsgebot}

9 Normativ im Vordergrund steht das sowohl in der Strafprozessordnung niedergelegte wie auch verfassungsund menschenrechtlich verankerte Beschleunigungsgebot. ${ }^{26}$ Danach obliegt es den Strafbehörden, die Verfahren unverzüglich an die Hand zu nehmen und sie ohne unbegründete Verzögerung zum Abschluss zu bringen (Art. 5 StPO). Dieser Verpflichtung zur zügigen Erledigung der Prozesse soll das abgekürzte Verfahren mit seinen schlanken Strukturen dienen. ${ }^{27}$ Dabei weitet sich freilich der ursprüngliche Bedeutungsgehalt der hervorgehobenen Maxime der Beschleunigung zu einem vieldeutigen Verständnis einer Verfahrenseffizienz aus. ${ }^{28}$ Gemeint ist nicht etwa bloss eine verzögerungsfreie Durchführung des Strafverfahrens zugunsten der beschuldigten Personen; vielmehr drängt eine «der Ressourcenschonung verpflichtete verfahrensökonomische Dimension» ${ }^{29}$ nach vorne, welche primär die Interessen der Strafbehörden an Zeit- und Kostenersparnis im Blick hat. ${ }^{30}$ Damit verbindet sich ein technokratisches Verständnis von Rechtsprechung, wonach die Strafbehörden bestrebt sind, die sie belastenden Prozessakten möglichst rasch aus ihrem Blickfeld zu entfernen, und zwar ohne vertiefte Reflexionen hinsichtlich einer Strafgerechtigkeit. ${ }^{31}$

25 Ausführlich LAGLER (Fn.12), S. 150 und 167 ff.; sodann THOMMEN (Fn.16), S.254ff.

26 Nachweise bei wolfgang woHLERs, in: Donatsch/Hansjakob/ Lieber (Hrsg.), Kommentar zur Schweizerischen Strafprozessordnung (StPO), 2. Aufl., Zürich et al. 2014, Art. 5 N1.

27 Vgl. z.B. NIKLAUS SCHMID / DANIEL JOSITSCH, Handbuch des schweizerischen Strafprozessrechts, 3. Aufl., Zürich et al. 2017, N145, und SABINE GLESS, Konsensuale Verfahrenserledigung in grösseren Wirtschaftsstrafverfahren - Chancen und Risiken, in: Ackermann/ Wohlers (Hrsg.), Umfangreiche Wirtschaftsstrafverfahren in Theorie und Praxis, Zürich et al. 2008, S.129.

28 WOLFGANG WOHLERS, Das Strafverfahren in den Zeiten der «Eil krankheit», NJW 2010, S. 2470, spricht von einem «multifunktionalen Instrument».

29 So für das deutsche Recht woHLERs, Eilkrankheit (Fn. 28), S. 2471.

30 Diese Einsicht sollte man nicht durch die euphemistische Behauptung überspielen, Ziel des abgekürzten Verfahrens sei «die Verwesentlichung der Justiz» (so aber ausdrücklich GEORGES GREINER, Schuld ohne Sühne? Am Beispiel des «plea bargaining» nach neuer StPO, fp 2009, S. 241).

31 Vergleichbare Tendenzen sind ebenfalls beim generellen Abbau der unmittelbaren Beweisaufnahme im ordentlichen Verfahren zu beobachten (dazu PETER ALBRECHT, Was bleibt von der Unmittelbar-

\section{b) Kurze Prozesse}

Konkret geht es da um eine (neue) «besondere» Verfah- 10 rensart, 32 die mit den Art.358-362 StPO eingeführt worden ist. Ihr materieller Gehalt wird durch die überwältigende Stosskraft des kriminalpolitischen Ziels einer Verfahrensvereinfachung und -beschleunigung geprägt. Das Konzept der Legislative zielt in erster Linie auf «radikale Beweisvereinfachungen» ${ }^{33} \mathrm{ab}$. Das abgekürzte Verfahren erweist sich insoweit als «kurzer Prozess» ${ }^{34}$, dessen Normierung lückenhaft und dürftig ausgestaltet ist. ${ }^{35}$ Es ist umhüllt von einem Schleier offener Fragen. Im Gesetz treten als äussere Kennzeichen in Erscheinung einerseits der stark reduzierte Umfang der behördlichen Ermittlungen und andererseits im Anschluss daran eine lediglich summarische richterliche Hauptverhandlung, die kaum ihren Namen verdient und zu einem Formalakt verkümmert. ${ }^{36}$ Gemäss ausdrücklicher Vorschrift (Art. 361 Abs. 4 StPO) findet vor Gericht kein Beweisverfahren statt, womit das Unmittelbarkeitsprinzip von vorneherein jegliche Relevanz einbüsst. Vor allem ist eine solide gerichtliche Überprüfung der zwischen den Parteien abgeschlossenen Vereinbarung nicht möglich. ${ }^{37}$ Viel mehr als eine unbefriedigende «Plausibilitätskontrolle» ${ }^{38}$ bleibt da nicht übrig. Die in Art. 30 Abs. 1 BV 39 statuierte richterliche Entscheidungskompetenz wird somit torpediert. Die Aufgabe der Justiz erschöpft sich in einer blossen Kontrollfunktion ohne eigenständige und unabhängige Rechtsfindung. ${ }^{40}$

keit?, ZStrR 2010, S. 190, und ARIANE NOSETTI-KAUFMANN, Strafbefehl, abgekürztes Verfahren und fehlende Unmittelbarkeit: Festhalten am Status quo - eine verpasste Chance?, ZStrR 2020, S. $253 \mathrm{ff.}$.).

32 Mit Recht kritisch gegenüber der Kategorisierung im Gesetz OBERHOLzER, Wahl des Verfahrens (Fn. 19), S. 14 und DERSELBE, Grundzüge (Fn.13), N1928, sowie THOMMEN (Fn.16), S. 241.

33 MARK PIETH, Schweizerisches Strafprozessrecht, 3. Aufl., Basel 2016, S. 249.

34 So deutlich THOMMEN (Fn.16), S.139f.; ähnlich auch OBERHOLZER, Grundzüge (Fn.13), N1971.

35 Zutreffend FELIX BOMMER, Kurzer Prozess mit dem abgekürzten Verfahren?, in: Heer (Hrsg.), Schweizerische Strafprozessordnung und Schweizerische Jugendstrafprozessordnung, Bern 2010, S.149f.; ferner WOLFGANG WOHLERS, Das abgekürzte Verfahren im schweizerischen Strafprozess, StV 2011, S. 570.

36 OBERHOLZER, Grundzüge (Fn.13), N1971; kritisch auch BOMMER, Abgekürztes Verfahren (Fn. 8), S.15.

37 Siehe KARL-LUDWIG KUNZ, Absprache und abgekürztes Verfahren nach künftigem Schweizerischem Strafprozessrecht, in: Jung/Luxenburger/Wahle (Hrsg.), Festschrift für Egon Müller, Baden-Baden 2008, S. 388 f. und 391.

38 HANS-HEINER KÜHNE, Strafprozessrecht, 9. Aufl., Heidelberg 2015, N749.3.

39 Bundesverfassung der Schweizerischen Eidgenossenschaft vom 18. April 1999 (BV; SR 101)

40 Im gleichen Sinne THommen (Fn. 16), S. 242f. - Demgegenüber erscheint die Behauptung eines Bundesstrafrichters, die «strategische Oberaufsicht» über die Anwendungspraxis des abgekürzten Verfahrens liege beim Gericht (so WALTER wÜTHRICH, Freier Markt beim Strafen?, AJP 2014, S. 1587) als überhöht. 
11 Das offenkundige Bestreben des Gesetzgebers nach Vereinfachungen hinterlässt tiefe Spuren. Es vermittelt ein unheimliches Kontrastprogramm im Vergleich zum ordentlichen Verfahren. Elementare Prinzipien des herkömmlichen Prozessrechts werden da-wie bereits zu Beginn erwähnt ${ }^{41}$ - partiell entwertet. Davon betroffen ist vor allem der Untersuchungsgrundsatz (Art. 6 StPO). ${ }^{42}$ Dies heisst, dass als Konsequenz des staatlichen Strafmonopols sowie des Verfolgungszwangs (Art. 7 StPO) ${ }^{43}$ den Strafbehörden die Ermittlung der materiellen Wahrheit ${ }^{44}$ obliegt.

12 Man kann zwar mit Recht daran festhalten, dass der erwähnte Grundsatz auch für das abgekürzte Verfahren gilt, weil das Gesetz keine Ausnahmeregelung vorsieht. ${ }^{45}$ Doch angesichts des Beschleunigungsdrucks auf die Strafbehörden verliert in der praktischen Umsetzung die Verpflichtung zur Ermittlung der materiellen Wahrheit an Substanz. Die Untersuchungstätigkeit wird unweigerlich auf ein Minimum zurückgefahren; denn andernfalls würde sie dem Ziel der besonderen Verfahrensart zuwiderlaufen. ${ }^{46}$ Als deren Folgen sind dann aber eklatante Abklärungsdefizite unübersehbar. Das liegt im Wesen der Absprachen zwischen den Parteien, das im Verzicht auf schwierige Beweisaufnahmen besteht, um komplexe Verfahren beschleunigt zu erledigen.

\section{c) Geständnis als Verfahrensvoraussetzung aa) Prozessualer Mittelpunkt}

13 Den Kern der Bemühungen um Effizienz in einem schlanken Verfahren bildet das Geständnis der Beschuldigten. Dieses erlangt eine herausragende Bedeutung in doppelter Hinsicht: Neben seiner strafmindernden Wirkung ${ }^{47}$ bildet es die vorrangige Verfahrensvoraussetzung. Das Gesetz fordert nämlich explizit, dass die beschuldigte Person «den Sachverhalt, der für die rechtliche Würdigung wesentlich ist, eingesteht [...]» (Art. 358 Abs.1 StPO). ${ }^{48}$ Das Geständnis steht - mit anderen Wor-

\footnotetext{
41 Vorne Ziff. I.2.

42 Botschaft zur Vereinheitlichung des Strafprozessrechts (Fn.12), S. 1294; ferner ausführlich LAGLER (Fn.12), S.124 ff., mit zahlreichen Nachweisen.

43 PIETH (Fn. 33), S. 43.

44 Statt vieler z.B. PIETH (Fn. 33), S. 43 und LAGLER (Fn. 12), S. 124 ff.; näher zur materiellen und forensischen Wahrheit wOHLERS (Fn. 26), Art. 6 N1f.; ferner ausführlich zum Ganzen CHRISTOPHER GETH, Fallstricke der Wahrheitsfindung im Vorverfahren - am Beispiel der beschuldigten Person, ZStrR 2020, S. $269 \mathrm{ff}$.

45 Z.B. GREINER/JAGGI (Fn.14), Art. 358 N19f.

46 Vgl. auch LAGLER (Fn.12), S.128f

47 Nachweise hinten Ziff. III.1. - KÜHNE (Fn. 38), N 750.1, betrachtet die durch Strafnachlass gewährte Belohnung des kooperativen Verhaltens im Verfahren als «Urform» der Absprache.

48 BOMMER, Abgekürztes Verfahren (Fn. 8), S. 11, nennt das «eine bestimmte Art von Geständnis».
}

ten - im prozessualen Mittelpunkt. ${ }^{49}$ Dabei gilt es zu beachten, dass die Strafbehörden Sachverhalte, die von den Beschuldigten eingestanden werden, nicht einfach als wahr hinnehmen dürfen, sondern diese zu «verifizieren» haben (Art.160 StPO). ${ }^{50}$

Abgesehen davon verdient hier neben dem Geständnis 14 der beschuldigten Personen deren Einlassung auf das abgekürzte Verfahren eine zusätzliche Beachtung. Der Beitrag zur beschleunigten Prozesserledigung beschränkt sich nicht auf die Anerkennung des Tatvorwurfs; vielmehr reicht die geleistete Kooperation deutlich weiter, indem die Beschuldigten sich aktiv an einer Vereinbarung mit der Staatsanwaltschaft beteiligen. ${ }^{51}$ Gleichzeitig wird auf diese Weise ihre Position als Prozesssubjekt geschwächt. Mit dem Verzicht auf ein ordentliches Verfahren ${ }^{52}$ verlieren die Tatverdächtigen den Schutz durch diese Verfahrensform. ${ }^{53}$ Damit sind bedeutsame Einschränkungen der Verteidigungsrechte verknüpft. Die Gesetzgebung erkennt darin einen positiv zu bewertenden Beitrag zur «Selbstverurteilung»54, was eine zwischen den Parteien ausgehandelte Strafminderung 55 rechtfertigen soll. ${ }^{56}$

\section{bb) Schuldvermutung}

Indem die gesetzliche Regelung für das abgekürzte Ver- 15 fahren ein Geständnis voraussetzt, stellt sie die rechtlich vorgegebene Unschuldsvermutung (Art.6Ziff. 2 EMRK57, Art. 6 BV und Art.10 Abs.1StPO) von vorneherein infrage und verkehrt sie in ihr Gegenteil, in eine Schuldvermu-

49 THOMMEN (Fn.16), S. 242; und ANDREAS DONATSCH, Das Geständnis im Strafbefehls- sowie im abgekürzten Verfahren, in: Kuhn/Margot/Aebi/Schwarzenegger/Jositsch/Donatsch (Hrsg.), Kriminologie, Kriminalpolitik und Strafrecht aus internationaler Perspektive, Festschrift für Martin Killias, Bern 2013, S.960.

50 LAGLER (Fn.12), S. 92 ff. und 124f.; sodann ausführlich VIKTOR LAUBE, Zu Tendenzen der schnellen Verfahrenserledigung. Das abgekürzte Verfahren gemäss Art. 358-362 der eidgenössischen Strafprozessordnung im Zuge des Beschleunigungsgebotes, Zürich et al. 2016, N339ff.

51 Zum deutschen Recht PETRA VELTEN, in: Wolter (Hrsg.), Systematischer Kommentar zur Strafprozessordnung, Bd. V, 5. Aufl., Köln 2016, vor \$§ 257b - 257c ff., Rdn. 11: «Kooperation und Verzicht auf Verteidigung».

52 Ausführlich LaUBE (Fn. 50), N901ff.

53 Siehe CHRISTA HAUSHERR, Das abgekürzte Verfahren in der schweizerischen Strafprozessordnung - Entlastung der Strafverfolgungsbehörden versus Rechtsstaatlichkeit, fp 2008, S.310f.

54 OBERHOLZER, Wahl des Verfahrens (Fn.19), S.15; ferner WINFRIED HASSEMER, Pacta sunt servanda - auch im Strafprozess? - BGH NJW 1989, 2270, JuS 1989, S. 892; ergänzend dazu THOMMEN (Fn.16), S. $277 \mathrm{ff}$.

55 Dazu hinten Ziff. III.

56 Hier erscheint eine gewisse Parallele zur gelegentlich erwähnten "Unterwerfungsgeste», die zu honorieren sei (siehe OBERHOLZER, Absprachen im Strafverfahren 1993 [Fn. 6], S. 17 und BOMMER, Abgekürztes Verfahren [Fn. 8], S.12).

57 Konvention zum Schutze der Menschenrechte und Grundfreiheiten (EMRK; SR 0.101). 
tung. ${ }^{58}$ Denn Verhandlungen über das Verfahrensergebnis machen nur dann einen Sinn, wenn man von der Täterschaft des Beschuldigten ausgeht.59 Das Aushandeln eines Sanktionsrabatts erweist sich als sinnwidrig, solange die Möglichkeit eines Freispruchs in Betracht kommt. Der gestellte Antrag (Art. 358 Abs.1 StPO) und die spätere Zustimmung zur Anklageschrift (Art.360 Abs. 4 StPO) sind als Verzicht auf die Unschuldsvermutung zu interpretieren. ${ }^{60}$ Dieses Faktum ist sehr ernst $\mathrm{zu}$ nehmen. Demzufolge bezeichnet Bommer das abgekürzte Verfahren, weil es nicht mit einem Freispruch enden kann, zutreffend als «Bestrafungsverfahren». 61

\section{cc) Sicherung des Schuldspruchs} digten lässt erahnen, dass die anvisierte Verfahrensstraffung sich nicht in einer Ressourcenschonung und einer zeitlichen Beschleunigung erschöpft. Denn der Strafverfolgung bietet die besondere Verfahrensart überdies eine (erwünschte) weitgehende Sicherung des Schuldspruchs (bzw. den Ausschluss eines Freispruchs ${ }^{62}$ ). Weil die Strukturen der abgekürzten Verfahren eine zuverlässige Überprüfung der Geständnisse von vorneherein faktisch verunmöglichen, ${ }^{63}$ haben geständige Beschuldigte regelmässig mit einer Verurteilung zu rechnen.

\section{d) Absprachen zwischen den Parteien \\ aa) Konsensuale Verfahrensverkürzungen}

17 Das Geständnis als Verfahrensvoraussetzung ist nicht als isoliertes Phänomen zu begreifen. Seine volle Tragweite lässt sich erst dann richtig erfassen, wenn man es als notwendigen Bestandteil des Deals betrachtet, der zwischen der beschuldigten Person und der Staatsanwaltschaft abgeschlossen wird. Die vom Gesetzgeber zwar nicht ausdrücklich erwähnte, aber dennoch einkalkulierte Absprache dient als probates Mittel der erwünschten Verfahrensbeschleunigung. Ganz offiziell und legal darf hier über den Ausgang des Prozesses verhandelt werden. Solchermassen wird «eine Art schweizerisches Plea-Bargaining-Verfahren» ${ }^{64}$ angestrebt. Es handelt sich um das Bemühen, «eine Verfahrensverkürzung auf konsensualer Grundlage» zu etablieren. ${ }^{65}$ Die

58 Siehe bspw. GREINER/JAGGI (Fn.14), vor Art. 358 - 362 N 43; unkritisch jedoch Zimmerlin (Fn. 7), N716 ff.

59 OBERHOLZER, Absprachen im Strafverfahren 1993 (Fn. 6), S.163f. und 173f.; sodann sehr deutlich aus der Sicht des deutschen Rechts HASSEMER (Fn. 54), S. 892: «... der Deal im Prozess lebt von einer Schuldvermutung».

60 BOMMER, Kurzer Prozess (Fn. 35), S. 165.

61 BOMMER, Abgekürztes Verfahren (Fn. 8), S. 31 und DERSELBE, Kurzer Prozess (Fn. 35), S. 165.

62 THOMmen (Fn.16), S. 243 sowie BomMER, Abgekürztes Verfahren (Fn. 8), S. 31 und DERSELBE, Kurzer Prozess (Fn. 35), S. 165.

63 Vgl. auch THOMMEN (Fn.16), S. 250 und KUNZ (Fn. 37), S. 392.

64 THOMMEN (Fn.16), S.149.

65 BOMMER, Abgekürztes Verfahren (Fn. 8), S. 9; siehe ergänzend dazu LAGLER (Fn.12), S.130 ff. erwähnten Absprachen beinhalten meistens Gegenleistungen der Staatsanwaltschaft, insbesondere eine mildere Sanktion für ein Geständnis der beschuldigten Person. Der klassische Fall des abgekürzten Verfahrens betrifft im Wesentlichen den Tausch eines Geständnisses gegen einen Sanktionsrabatt. 66

\section{bb) Kompensation von Wahrheitsdefiziten?}

Angesichts der dominanten konsensualen Komponen- 18 ten in der verkürzten Strafuntersuchung fragt sich ernsthaft, ob die feststellbaren Abklärungsmängel im Vergleich zum ordentlichen Verfahren auf diese Weise aufgewogen werden können. ${ }^{67}$ Diesbezüglich ergeben sich erhebliche Zweifel mit Blick auf den Untersuchungsgrundsatz und das Prinzip der materiellen Wahrheit. In den Vordergrund tritt die schwer zu leugnende Erkenntnis: «Absprachen kompensieren keine Wahrheitsdefizite.» ${ }^{68}$ Deshalb fällt das Konsensprinzip für die Legitimation «defizitärer Verfahren» ausser Betracht. ${ }^{69}$ Dabei ist vor allem zu beachten, dass die für die Verfahrenseinleitung so bedeutsamen Geständnisse regelmässig taktischer Natur und folglich unter dem Gesichtspunkt der materiellen Wahrheit unzuverlässig sind .70 Die Kritik, das abgekürzte Verfahren sei durch eine «gewissermassen ausgehandelte Wahrheit» ${ }^{71}$ geprägt, lässt sich nicht in Abrede stellen. Das Konsensprinzip und die behördliche Ermittlungspflicht gemäss Art. 6 StPO schliessen sich gegenseitig aus. ${ }^{72}$

\section{e) Eigenständiger Prozess}

Die in Ziff. II.2 erfolgte nähere Charakterisierung des ab- 19 gekürzten Verfahrens als «kurzer Prozess» führt - das lässt sich rückblickend und zusammenfassend festhalten - die neuere wissenschaftliche Debatte auf die Spur eines eigenständigen Prozesses. ${ }^{73}$ Innerhalb des Strafprozessrechts nimmt die neue Verfahrensform nämlich eine Art Zwischenstellung ein und steht folglich im Konflikt zu den herkömmlichen Rechtsprinzipien. Dem ordentlichen Verfahren obliegen seiner Idee nach vor allem die strikte Beachtung des Untersuchungsgrund-

66 Zuweilen auch als «eine Art Ablasshandel» denunziert (KONRAD JEKER, Abgekürzte Verfahren im Wirtschaftsstrafprozess, in: Ackermann/Hilf (Hrsg.), Kurzer Prozess, zu kurzer Prozess - im Wirtschaftsstrafverfahren, Zürich et al. 2019, S.118).

67 THOMMEN (Fn.16), S. 260 ff.

68 LAGLER (Fn.12), S.130; ebenso THOMMEN (Fn.16), S. $263 \mathrm{f}$.

69 VELTEN (Fn. 51), vor \$\$257b-257cff., Rdn. 11.

70 THOMMEN (Fn.16), S. 263.

71 DANIEL JOSITSCH / PATRICK BISCHOFF, Das Abgekürzte Verfahren gemäss Art. 365-369 des Entwurfs zu einer Schweizerischen Straf prozessordnung, in: Niggli/Hurtado Pozo/Queloz (Hrsg.), Festschrift für Franz Riklin, Zürich et al. 2007, S. 433; zustimmend LAG LER (Fn.12), S.132; ähnlich auch OBERHOLZER, Wahl des Verfahrens (Fn.19), S.15: «konsensuale Wahrheit».

72 WOHLERS, Eilkrankheit (Fn. 28), S. 2474.

73 So vor allem THOMmen (Fn.16), S. $241 \mathrm{f}$. und im Anschluss daran oBERHOLZER, Wahl des Verfahrens (Fn.19), S.15ff. 
satzes (Art. 6 StPO) und in der Folge davon die Ermittlung der materiellen Wahrheit. ${ }^{74}$ Das abgekürzte Verfahren hingegen ist im Vergleich dazu äusserlich stark abweichend ausgestaltet; überdies verfolgt es inhaltlich (teilweise) auch eine andere Zielsetzung. Denn es geht ihm zur Hauptsache «um eine möglichst reibungslose, kosten- und zeitarme Liquidation der durch die Tat ausgelösten Konfliktsituation». ${ }^{75}$ Die materielle Wahrheit wird in den Hintergrund gedrängt, und stattdessen tritt als Legitimationsgrundlage der Bestrafung «ausschliesslich und allein die konsensuale Einwilligung des Betroffenen» in den Vordergrund. ${ }^{76}$

Eine solche Optik kann dazu verführen, die rechtsstaatlichen Bedenken gegen die kurzen Prozesse zu verharmlosen. Derzeit kommt aus der Politik ein permanenter und nachhaltiger Druck für eine verfahrensmässige Entlastung der Strafbehörden in der Form schlanker Strukturen. Trotzdem ist seitens der Gesetzgebung Zurückhaltung geboten im Hinblick auf derartige Begehren einer Entformalisierung. Namentlich besteht die Gefahr, dass die legitimatorische Potenz von Kategorien wie Zustimmung und Konsens überhöht wird. Zweifel erweckt die Annahme, die Zustimmung zum abgekürzten Verfahren könne die Abweichung von Verfahrensstandards rechtfertigen. ${ }^{77} \mathrm{Zu}$ warnen ist vor einer Geringschätzung der Schutzfunktionen des ordentlichen Verfahrens zugunsten der Beschuldigten. Diese heikle kriminalpolitische Problemstellung lässt sich gerade für das hier schwerpunktmässig zu behandelnde Thema der Sanktionsrabatte gut veranschaulichen (zunächst in Ziff. III und sodann noch grundsätzlicher in Ziff. IV).

\section{Das ausgehandelte Sanktionsmass}

\section{Die gesetzlichen Vorgaben}

21 Die Art. 358 ff. StPO schweigen sich darüber aus, nach welchen inhaltlichen Kriterien die beteiligten Prozessparteien in ihren Absprachen die jeweilige Strafhöhe festzusetzen haben. Hierfür mangelt es offenkundig an einer ausdrücklichen Norm. Gewisse Anhaltspunkte lassen sich lediglich indirekt aus der Gesetzgebung ableiten. So schreibt Art. 362 Abs. 1 lit. c StPO vor, dass im Bestätigungsverfahren das Gericht darüber zu befinden habe, «ob die beantragten Sanktionen angemessen sind». Der Begriff «angemessen» wird in Anlehnung an die bundesrätliche Botschaft regelmässig im Sinne einer

\footnotetext{
74 Vgl vorne Ziff. II.2.b.

75 THOMMEN (Fn. 16), S. 242; zustimmend OBERHOLZER, Wahl des Verfahrens (Fn.19), S.16.

76 OBERHOLZER, Wahl des Verfahrens (Fn. 19), S.15.

77 Siehe WOHLERS, Das abgekürzte Verfahren (Fn. 35), S. 572 und ANDREAS DONATSCH, Das schweizerische Strafprozessrecht, SJZ 2004, S.327.
}

Schuldangemessenheit verstanden. ${ }^{78}$ Daraus resultiert dann die vorherrschende Ansicht, wonach die allgemeinen im Strafgesetzbuch niedergelegten Regeln der Strafzumessung ebenfalls für das abgekürzte Verfahren gelten. ${ }^{79}$ Das betrifft unter anderem auch die strafmindernde Wirkung des Geständnisses, die durch das Bundesgericht ${ }^{80}$ wie auch die überwiegende Doktrin ${ }^{81}$ unter bestimmten Voraussetzungen anerkannt wird.

Diegeforderte pauschale Übernahme der für den ordent- 22 lichen Prozess massgebenden Strafzumessungskriterien ist keineswegs zwingend und in der Sache nicht unproblematisch. Es scheinen nämlich Bedenken auf, ob die rechtspolitische Konzeption des abgekürzten Verfahrens mit der unmittelbar an die Tatschuld anknüpfenden Strafzumessungsvorschrift des Art. 47 StGB kompatibel ist. Diesbezüglich muss man in erster Linie an das Effizienzinteresse erinnern, das sich mit schlanken Verfahren eng verbindet und auf das Strafmass durchschlägt. Erkennbar wird hier die Strafminderung als Folge eines positiv bewerteten Verhaltens während der behördlichen Ermittlungen. Unter einem solchen Blickwinkel dominieren während der Verhandlungen zwischen den Parteien faktisch nicht die Schwere der begangenen Straftaten, sondern das Prozessverhalten des Tatverdächtigen sowie das gemeinsame Bestreben, einen für die Staatsanwaltschaft wie auch die beschuldigte Person akzeptablen Deal hinsichtlich der Strafsanktion zu erreichen. Wegleitend ist demnach primär oder zumindest ganz wesentlich der prozessökonomische Nutzen der ausgehandelten Strafe und nicht (allein) das Gewicht der Tatschuld.

In der Konsequenz führt das - wie die Praxis lehrt - zu 23 tendenziell milden Sanktionen, weil diese den Anreiz erhöhen, dass die beschuldigten Personen sich auf einen kurzen Prozess einlassen. ${ }^{82}$ Eine solche Privilegierung der Kooperationsbereitschaft erweckt im Hinblick auf den Grundsatz der Rechtsgleichheit (Art. 8 BV) Bedenken; denn Beschuldigte in besonders komplexen Fällen

78 Botschaft zur Vereinheitlichung des Strafprozessrechts (Fn.12), S.1296: «dem Verschulden angemessen»; zustimmend GIGER (Fn. 5), S. 75 und SCHWARZENEGGER (Fn.14), Art. $362 \mathrm{~N} 5$.

79 In diesem Sinne etwa GREINER/JAGGI (Fn.14), Art. 362 N14; ferner ausführlich E.JAGGI (Fn. 2), S. $48 \mathrm{ff}$.

80 Z.B. Urteil des Bundesgerichts 6B 1248/2017, 6B 1278/2017 vom 21. Februar 2019 E 7.5.4; im gleichen Sinne auch Urteil des Bundesgerichts 6B_282/2018 vom 24. August 2018 E 2.6 und Urteil des Bundesgerichts 6B_312/2016 vom 23.Juni 2016 E1.3.2.

81 Vgl. etwa GÜNTER STRATENWERTH / FELIX BOMMER, Schweizeri sches Strafrecht, Allgemeiner Teil II, 3. Aufl., Bern 2020, § 5 N 52 ff., und HANS WIPRÄCHTIGER / STEFAN KELLER, in: Niggli/Wiprächtiger (Hrsg.), Basler Kommentar, Strafrecht I, 4. Aufl., Basel 2019, Art. 47 N169ff.

82 Ob die Wahl des abgekürzten Verfahrens neben der Strafminderung zufolge des Geständnisses darüber hinaus zu einer weitergehenden Reduktion der Strafe führen darf, wird in der Wissenschaft kontrovers diskutiert (dazu GIGER [Fn. 5], S. 76, mit weiteren Hinweisen). 
können eher als andere Tatverdächtige mit einem abgekürzten Verfahren samt entsprechender Strafreduktion rechnen. Wenn nun aber die Verfahrensschwierigkeit indirekt sich als Strafzumessungsfaktor erweist, verletzt das die «Strafrechtsgleichheit». 83

\section{Der Bemessungsvorgang}

\section{a) Sentence Bargaining} Strafbemessung bei der erwähnten besonderen Prozessform durch spezifische Eigenheiten kennzeichnet. So setzt im ordentlichen Verfahren ein unabhängiges Gericht anlässlich einer öffentlichen Hauptverhandlung die jeweilige Strafhöhe autoritativ fest. Demgegenüber herrscht im abgekürzten Verfahren ein konsensualer Akt vor. Das Strafmass wird nämlich zwischen der Staatsanwaltschaft und der beschuldigten Person im Rahmen einer Vereinbarung ausgehandelt (Sentence Bargaining), dies heimlich unter Ausschluss der Öffentlichkeit. Allein schon die knappen Hinweise genügen, um den ganz erheblichen Unterschied zwischen der Verhandlungssituation im Abspracheverfahren und der Urteilsberatung innerhalb des richterlichen Gremiums nach durchgeführter Beweisaufnahme zu belegen. abgekürzten Prozess nicht präsent; es wirkt an der Festlegung des konkreten Strafmasses nicht eigenständig mit. Ihm bleibt lediglich die untergeordnete Aufgabe, die durch die Parteien vereinbarte Sanktion in einer summarischen Verhandlung auf ihre Angemessenheit zu überprüfen (Art. 362 Abs. 1 lit. c StPO). ${ }^{84}$ Somit steht die ideale strafrechtliche Zielsetzung einer gerechten und angemessenen Schuldvergeltung von vorneherein in Gefahr; gleichzeitig rückt der Aspekt der Rechtsgleichheit in weite Ferne.

\section{b) Begrenzte richterliche Überprüfung}

wischen den Parteien getroffene Absprache ist undurchsichtig; von aussen her lässt sich nur schwer erkennen, welche Kriterien das Ergebnis jeweils beeinflussen. Ohne Durchführung eines Beweisverfahrens (Art. 361 Abs. 4 StPO) ist es ausgeschlossen, dem vorgegebenen komplexen normativen Programm gerecht zu werden. Auf welche Weise sollen denn in einem Genehmigungsverfahren die Richterinnen und Richter beispielsweise die subjektive Tatseite oder die Täterkomponenten (gemäss Art. $47 \mathrm{StGB}$ ) feststellen und beurteilen? Wie wollen sie ferner einschätzen, ob die von der Staatsanwaltschaft für das kooperative Verhalten der Beschuldigten angebotene Belohnung im Rahmen des Deals

83 So deutlich VELTEN (Fn. 51), vor \$§257b-257c ff., N19a. 84 OBERHOLZER, Grundzüge (Fn.13), N1979f. sachlich angebracht ist? Schliesslich lässt sich die Angemessenheit des vereinbarten Rabatts im konkreten Fall allein schon aus praktischen Gründen nicht mit der gebotenen Seriosität überprüfen. ${ }^{85}$ Denn das Gericht stützt seinen Befund im Wesentlichen auf die Verfahrensakten, die üblicherweise nur spärliche Informationen zu den strafmassrelevanten Daten enthalten. Das Abspracheergebnis verharrt somit weitgehend im Dunkeln. Daran vermag auch die häufig geforderte Pflicht zur Dokumentation der Absprache ${ }^{86}$ nur wenig zu ändern.

\section{Das Risiko unangemessener Rabatte}

Die gesetzliche Regelung öffnet einer willkürlichen 27 Sanktionierung unvermeidbar Tür und Tor. Insbesondere sind hier aus vorwiegend prozessökonomischer Motivation kräftige Rabatte, die unverantwortlich milde Sanktionen bewirken, nicht auszuschliessen. Dass es letztlich das Gericht sei, welches über die Strafzumessung entscheide, ${ }^{87}$ lässt sich kaum ernsthaft behaupten. ${ }^{88}$ Vielmehr findet ein Bedeutungsverlust der unabhängigen Justiz statt. 89

Abgesehen davon fragt sich, wem im abgekürzten Ver- 28 fahren eine strikt nach den Prinzipien des materiellen Rechts ausgerichtete Bemessung der Strafhöhe überhaupt ein echtes Anliegen ist. Von einem Sanktionsrabatt profitieren nämlich mehr oder minder alle Verfahrensparteien; ${ }^{90}$ auch die Gerichte sind letztlich froh, wenn die Fälle möglichst reibungslos vom Tisch gehen. Von den beteiligten Personen hat folglich niemand ein eminentes Interesse an einer wirksamen richterlichen Kontrolle der getroffenen Vereinbarung. Ebenso wenig erstaunt denn auch die allgemeine (jeweils nicht näher begründete) Ansicht, wonach bei der Ablehnung der beantragten Sanktion grosse Zurückhaltung geboten sei ${ }^{91}$. So wird denn etwa die Ansicht vertreten, dass «die Genehmigung wegen der Sanktionen nur dann verweigert werden» sollte, wenn «diese geradezu rechtmissbräuch-

85 Ähnlich auch THOMmEN (Fn.16), S.199f.

86 So z.B. GREINER/JAGGI (Fn.14), Art. 358 N62 ff., mit weiteren Nachweisen.

87 So GREINER/JAGgi (Fn.14), Art.362 N14; ebenso E.JAGGi (Fn. 2), S. 49; ferner LAUBE (Fn. 50), N 442.

88 Mit Recht hält BOMMER, Abgekürztes Verfahren (Fn. 8), S. 108 fest, dass der Ausspruch der Strafe «vom Gehalt her ein «Urteil» der Staatsanwaltschaft» darstellt; ebenso тноммEN (Fn.16), S. 228.

89 Ähnlich auch BOMmER, Abgekürztes Verfahren (Fn. 8), S.15; ebenfalls kritisch LAUBE (Fn. 50), N 443. - Der geäusserte Befund ist nicht allein ein Kennzeichen des abgekürzten Verfahrens, sondern widerspiegelt heute einen allgemeinen (höchst bedenklichen) Trend in der Entwicklung des Strafprozessrechts (dazu PIETH [Fn.33], S. 250f. und ALBRECHT, Strafrecht [Fn. 21], S. 393 f.).

90 Siehe OBERHOLZER, Grundzüge (Fn.13), N1970.

91 Nachweise bei LAGLER (Fn. 12), S.114; siehe sodann IRMA JAGGI, Die strafprozessuale Absprache vor den Schranken des Gerichts. Rolle und Einfluss des Gerichts im abgekürzten Verfahren, Zürich et al. 2016, S. 185 . 
lich hoch oder tief ausgefallen sind».92 Infolgedessen wird in der Praxis ein vereinbartes Strafmass nur äusserst selten durch das Gericht zurückgewiesen. ${ }^{93}$ Offenkundig verdrängen also die prozessualen Vereinfachungs- und Beschleunigungsinteressen die Bestrebungen nach einem (schuld)gerechten Strafmass.

\section{Die hybride Sanktionierung}

Eine Analyse der soeben angestellten Überlegungen lässt unschwer erkennen, dass das abgekürzte Verfahren im Effekt auf eine hybride Sanktionierung von Straftaten hinausläuft. Für die Festlegung der Strafhöhe wird zwar verbal die Tatschuld (ergänzt durch präventive Komponenten) als zentrales Kriterium hervorgehoben. Andererseits aber fliessen zugleich auch ziemlich ungehemmt Bedürfnisse der Verfahrenseffizienz in das Strafmass ein. Demnach findet die Kombination einer materiell-rechtlichen mit einer prozessualen Basis statt. In diesem zwitterhaften Konstrukt bleibt indes schon theoretisch unklar, wie die verwendeten Massstäbe, die aus unterschiedlichen Bezugssystemen stammen, in kontrollierbarer Weise miteinander zu verrechnen sind. Handelt es sich hier nicht um letztlich inkommensurable Grössen?

Die zuletzt angesprochenen Unsicherheiten führen auf eine sanktionstheoretische Ebene, wo Skrupel auftauchen, ob es sich bei der in einem Deal vereinbarten Sanktion wirklich um eine «Strafe» im herkömmlichen Sinne handelt. Die Institution des abgekürzten Verfahrens ist jedenfalls Ausdruck eines Wandels im Verständnis des Strafverfahrens wie auch der Strafsanktionen. Die zwischen den Parteien abgesprochene Strafe erscheint als prozessual verkümmerte Strafe, weil sie aus einem defizitären Verfahren hervorgeht und das schuldangemessene Mass durch Bedürfnisse der Verfahrenseffizienz reduziert ist. Unter diesem Blickwinkel ist es kein Zufall, dass gerade die Betäubungsmitteldelikte mit ihrer brüchigen verfassungsrechtlichen und kriminalpolitischen Legitimation ${ }^{94}$ im abgekürzten Verfahren eine hohe Relevanz aufweisen. Offenkundig verblassen hier die klassischen Strafzwecke und somit auch der pönale Charakter der staatlichen Reaktionen auf die begangenen Normverletzungen. Zurück bleiben etwas ziellose Sanktionen, die durch den Willen zum raschen Konsens durchdrungen werden.

\footnotetext{
92 DIEGO R. GFELLER, Nachverhandlungen im abgekürzten Strafverfahren?, AJP 2017, S. 591; im gleichen Sinne YVAN JEANNERET / ANDRÉ KUHN, Précis de procédure pénale, 2 éd., Berne 2018, N17075; und LIPS-AMSLER (Fn.15), S.148f.; abweichend hingegen LOÏC PAREIN, La négociation de la peine dans le cadre de la procédure simplifiée, Jusletter vom 29. November 2010, N27.

93 Ausführlich I. JAGGI (Fn. 91), S.182 ff.

94 Ausführlich dazu PETER ALBRECHT, Die Strafbestimmungen des Betäubungsmittelgesetzes (Art.19-28l BetmG), 3. Aufl., Bern 2016, Einleitung, N34ff.
}

\section{Der Zwang zur Kooperation}

\section{Das Nemo-tenetur-Prinzip}

Die regelmässig zwischen den Parteien vereinbarte 31 Sanktion ist mit der im Gesetz verankerten Geständnisvoraussetzung95 unmittelbar verknüpft: Ohne eingestandenen Tatvorwurf finden keine Verhandlungen über die Strafhöhe statt. Demzufolge ist die beschuldigte Person im prozessualen Verhalten beschränkt; ihre Handlungsoptionen sind limitiert. Die Doktrin spricht (mit kritischem Unterton) von einer «Art von institutionalisiertem Geständnisdruck» ${ }^{96}$. Dieser stellt neben der Unschuldsvermutung ebenso das Nemo-tenetur-Prinzip infrage, ${ }^{97}$ das aus übergeordnetem Recht stammt und für den Gesetzgeber verbindlich ist. ${ }^{98} \mathrm{Hinzu}$ kommt noch, dass beim abgekürzten Verfahren die Bereitschaft zur Zusammenarbeit mit den Strafbehörden sich nicht in einem Geständnis erschöpft. Sie reicht deutlich weiter, indem die Beschuldigten sich auf die besondere Verfahrensform einlassen und aktiv an der Erledigung teilnehmen. Solchermassen verzichten sie gleichzeitig auf die schützenden Formen des ordentlichen Verfahrens, d.h. insbesondere auf einen Teil der ihnen zustehenden Verteidigungsrechte.

Der angesprochene Konflikt mit der Selbstbelastungsfrei- 32 heit tritt umso markanter in Erscheinung, je grösser die «Sanktionsschere» ${ }^{\mathbf{9} 9}$, d.h. der zu erwartende Rabatt (im Vergleich zum ordentlichen Prozess), ausfällt, den die Staatsanwaltschaft für ein kooperatives Verhalten in Aussicht stellt. Im Ergebnis bedeutet dies (umgekehrt), dass für diejenigen Personen, die sich auf das abgekürzte Verfahren nicht einlassen (wollen), eine entsprechendeallenfalls erhebliche - Strafminderung entfällt. ${ }^{100}$ Insoweit droht ihnen eine Benachteiligung im Vergleich $\mathrm{zu}$ den geständigen und mitwirkungsbereiten Beschuldigten. Das kann tatverdächtige Personen leicht dazu veranlassen, auf ihr gesetzliches Schweigerecht zu verzichten, ein Geständnis abzulegen und der seitens der Staatsanwaltschaft eröffneten Anklageschrift zuzustimmen (Art. 360 Abs. 4 StPO). Die Betroffenen befinden sich

\section{Siehe vorne Ziff. II.2.c.}

96 HANS VEST, Amerikanisierung des Schweizerischen Strafprozes ses?, in: Meier-Schatz/Schweizer (Hrsg.), Recht und Internationalisierung, Zürich 2000, S. 302; ebenso JOSITSCH/BISCHOFF (Fn. 71), S. 435 .

97 GLESS (Fn. 27), S. 133.

98 Dazu MARC THOMmen, Darf der Beschuldigte im Strafverfahren lügen?, sui generis 2018, S. $316 \mathrm{ff}$., mit zahlreichen Nachweisen; ferner WOHLERS, Das abgekürztes Verfahren (Fn.35), S. $572 \mathrm{f}$.

99 KÜHNE (Fn. 38), N748.1.

100 Vgl. PETER ALBRECHT, Schweigen im Strafprozess, in: von Sass (Hrsg.), Stille Tropen, Freiburg et al. 2013, S.148; siehe ebenfalls STRATENWERTH/BOMMER (Fn. 81), §5 N57; und ARZT (Fn. 30), S. 239 . 
dabei in einem mehr oder minder ausgeprägten Zwang, weil sie in ihrer Willensentschliessung und -betätigung nicht wirklich frei sind.

Andererseits ist zu bedenken, dass in jedem Strafprozess die Freiheit, Entscheidungen zur eigenen Verteidigung zu treffen, ohnehin begrenzt ist. ${ }^{101}$ Die den Strafbehörden von der Gesetzgebung zur Verfügung gestellten Zwangsmittel und Sanktionen üben auf alle Tatverdächtige von vorneherein unvermeidbar einen gewissen (systemimmanenten) Druck aus. Das jeweilige Aussageverhalten ist insoweit erheblich durch den Rahmen der Verfahrensordnung geleitet. Das offenbart sich gerade auch im ordentlichen Verfahren besonders augenfällig beim Recht, die Aussage zu verweigern (Art.113 Abs. 1 StPO). Das genannte Schweigerecht erweist sich nämlich als durchaus zwiespältig, weil dessen Wahrnehmung für die beschuldigte Person mit Blick auf ihre Verteidigungsmöglichkeiten unter Umständen negative Folgen entfalten kann. ${ }^{102}$ Eine solche Beschränkung der prozessualen Entscheidungsfreiheit erlangt aber im abgekürzten Verfahren ein noch deutlich erhöhtes Gewicht wegen des zumeist in Aussicht stehenden Sanktionsrabatts. Die geschürte Erwartung einer milderen Sanktion lässt niemanden unbeeinflusst, zumal die ohne Absprache drohende Strafe als Alternative zum Voraus kaum bezifferbar ist. ${ }^{103}$ Der dadurch bewirkte qualifizierte Druck auf die beschuldigte Person mit dem Ziel, sie zu einer Mitwirkung im Verfahren zu bewegen, weist auf eine unübersehbare rechtsstaatliche Problematik hin.

\section{Die verbotenen Zwangsmittel}

in, dass der feststellbare Druck nicht bloss durch die Gesetzgebung (namentlich durch das Geständniserfordernis in Art. 358 Abs. 1 StPO) verursacht ist, sondern zusätzlich durch das Verhalten der Staatsanwaltschaft anlässlich des Deals hervorgerufen wird. Gemeint ist deren Angebot eines Rabatts auf der Sanktionsebene im Gegenzug zur Bereitschaft des Beschuldigten, im Verfahren zwecks Vereinfachung und Beschleunigung mit der Behörde zu kooperieren. Dabei bleibt - quasi als Konnex zur Selbstbelastungsfreiheit -zu beachten, dass die Strafverfolgungsinstanzen das Einverständnis der Mit-

101 LAGLER (Fn. 12), S.139f. und HAUSHERR (Fn. 53), S. 312.

102 Allgemein zur «Ambivalenz der Aussageverweigerung» ALBRECHT, Schweigen (Fn.100), S. 147 ff.

103 Siehe HAUSHERR (Fn. 53), S. 312. wirkung nicht mittels verbotener Beweiserhebungsmethoden (Art.140 StPO) erlangen dürfen. ${ }^{104}$ Durch dieses Verbot, beispielsweise Drohungen oder Versprechungen einzusetzen, sollen die Tatverdächtigen vor unzulässigem Zwang geschützt werden.

Das wird im Grundsatz zwar generell anerkannt, doch 35 im Einzelfall ist es oft schwierig, zwischen der erlaubten und unerlaubten behördlichen Einwirkung eine präzise Grenze zu ziehen. ${ }^{105}$ Für das abgekürzte Verfahren gestaltet sich die Ausgangslage als besonders anspruchsvoll, weil das Strafmass zwischen den Parteien jeweils unter Ausschluss der Öffentlichkeit ausgehandelt wird. Unter diesen Umständen ist eine wirksame Kontrolle der vereinbarten Sanktionen auf ihre Schuldangemessenheit von vorneherein ausgeschlossen (und zwar selbst seitens des Gerichts in seinem Genehmigungsentscheid gemäss Art. 362 Abs. 1 StPO). Wer kann denn da schon seriös überprüfen, ob und in welchem Ausmass eine beschuldigte Person beim Einverständnis mit dem angebotenen Rabatt unter unzulässigem Druck stand?

\section{Schlussfolgerungen}

Die geschilderte Sachlage verdeutlicht, dass bei jedem 36 Deal um die Strafsanktion mit der Möglichkeit eines Verstosses gegen das elementare Recht der Selbstbelastungsfreiheit ernsthaft zu rechnen ist; dieses Risiko lässt sich in keinem Fall ausschliessen. Eine solchermassen negative Erkenntnis stellt das abgekürzte Verfahren fundamental infrage und führt letztlich zur Forderung nach einem Verzicht auf die (grosszügigen) Sanktionsrabatte. Das ziemlich radikale Postulat tangiert die erwähnte Verfahrensart allerdings in ihrem Kernanliegen. Denn ohne Angebot einer im Vergleich zum ordentlichen Prozess spürbar milderen Sanktion sind verfahrenserleichternde Absprachen zwischen der Staatsanwaltschaft und den beschuldigten Personen in der Regel nur schwer vorstellbar. Demnach mag die meinerseits geäusserte Skepsis gegenüber der Strafzumessungspraxis und dem damit verknüpften Kooperationszwang einen Anlass bieten, die kriminalpolitische Legitimation des durch Art. 358-362 StPO geregelten Abspracheverfahrens insgesamt gründlich zu überdenken.

104 Siehe LAGLER (Fn. 12), S. 138, mit Nachweisen.

105 Einzelheiten bei SABINE GLESS, in: Niggli/Heer/Wiprächtiger (Hrsg.), Basler Kommentar, Schweizerische Strafprozessordnung, 2. Aufl., Basel 2014, Art.140 N 37 ff.; LAGLER (Fn. 12), S.139ff.; HAUSHERR (Fn. 53), S.311ff. 